\title{
An exploration of key barriers to healthcare providers' use of food prescription (FRx) interventions in the rural South
}

\author{
Kara Beth Coward ${ }^{1}$, Anne Cafer ${ }^{1,2, *}$, Meagen Rosenthal ${ }^{2,3}$, David Allen III $^{3}$ and \\ Queenie Paltanwale ${ }^{3}$ \\ 'Department of Sociology and Anthropology, The University of Mississippi, 543 Lamar Hall, PO Box 1848, Oxford, \\ MS 38677-1848, USA: ${ }^{2}$ Community First Research Center for Wellbeing and Creative Achievement (CREW), \\ The University of Mississippi, Oxford, MS, USA: ${ }^{3}$ Department of Pharmacy Administration, School of Pharmacy, \\ The University of Mississippi, Oxford, MS, USA
}

Submitted 3 September 2020: Final revision received 14 December 2020: Accepted 21 December 2020: First published online 11 January 2021

\begin{abstract}
Objective: The purpose of this exploratory study was to examine the attitude towards food prescriptions (FRx) interventions among clinicians and identify potential barriers to their use in clinical practice.

Design: The current study employed an exploratory research design using in-depth semi-structured interviews. Research participants were selected from primary care facilities, family practice offices and obesity clinics located in Mississippi and Louisiana. Setting: Providers selected for participation in the current study serve predominantly rural, low-income communities in the US South.

Participants: From an original population of fifty healthcare providers that included physicians, registered dieticians and nurse practitioners, from Oxford, Tupelo, Batesville, Jackson, and Charleston, MS and New Orleans, LA. Fifteen healthcare providers agreed to participate, including three physicians, four registered dieticians, three nurses and three nurse practitioners.

Results: The current study found that while healthcare providers expressed a desire to use FRx interventions, there was a universal lack of understanding by healthcare providers of what FRx interventions were, how they were implemented and what outcomes they were likely to influence.

Conclusions: The current study identified key bottlenecks in the use of FRx interventions at the clinic level and data provided evidence for two key recommendations: (1) development and validation of a screening tool to be used by clinicians for enrolling patients in such interventions and (2) implementation of nutrition education in primary professional training, as well as in continuing education.
\end{abstract}

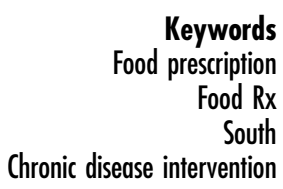

Current healthcare literature generally identifies fruit and vegetable food prescriptions (FRx) as a viable clinical intervention for improving patients' dietary consumption patterns, improving patient health outcomes and aiding in the prevention or management of nutrition-related chronic diseases, such as obesity, diabetes and CVD. While there is no universal protocol or standard of practice specific to FRx, for the purposes of the current study, based on existing interventions and the literature, the authors define FRx as a bealth focused intervention used by clinical bealthcare providers to encourage their patients to improve their dietary consumption patterns by increasing patient access to bealthier foods and improving their nutritional literacy ${ }^{(1-19)}$. An emphasis on food access for food insecure individuals and disease management are also important provisions in FRx interventions. These interventions typically include a clinical provider writing out a traditional prescription but for quantities of fruits and vegetables rather than medications ${ }^{(1-19)}$. This prescription is then redeemed in a variety of ways - vouchers at a local farmers market, electronic benefit transfer (EBT) card at a local grocer or retailer or through a mobile market ${ }^{(1-19)}$. Each of these methods is designed to add money to the household food budget, specifically for fresh fruits and vegetables. Eligibility for these interventions ranges from poor food security status to the presence of a chronic health conditions (obesity, diabetes, pre-diabetes) ${ }^{(1-19)}$.

FRx interventions can have substantial short- and longterm effects on patient health, aiding in the management of chronic conditions, in the prevention of diseases and 
reducing long-term health costs ${ }^{(13-14)}$. Patients with diabetes, in particular, have been targeted in FRx programming. FRx interventions, originally created to benefit patients with diabetes, were eventually expanded to benefit all individuals in need. This expansion resulted in improved access to fresh fruits and vegetables for households, often living in poor food environments and systemically addressed health inequalities in low socio-economic status (SES) communities ${ }^{(11,19)}$. Others have documented the role FRx interventions play in reducing diabetes patients' HbA1c levels and subsequently increasing their healthcare savings by up to $\$ 24000$ per year ${ }^{(12)}$. While many FRx interventions target those with nutrition-related chronic conditions, others have included those with stroke, cancer, coeliac disease, metabolic disease and women who are pregnant ${ }^{(12,15-17,20-24)}$. Despite this evidence, the expansion of FRx interventions into mainstream primary care practice has been slow and uneven.

As currently implemented in the USA, FRx interventions rarely recruit through a medical provider but often rely on poor health or presence of a medical condition to determine eligibility ${ }^{(1-18)}$. Yet, studies that have evaluated interventions incorporating clinicians indicate that prescriptions supplied by physicians are generally more highly valued by patients because of the symbolic power and patientperceived authority of the recommendation ${ }^{(10)}$. This presents a particular dilemma for practitioners - knowing that clinics are powerful resources in the implementation and effectiveness of FRx interventions, but not understanding the lack of clinic level adoption or involvement.

The examination of this literature highlights a number of potential barriers to FRx adoption by clinical teams. The first is that there is no standard of practice around implementation. Specifically, there is a wide range but no universally accepted method of screening patients for eligibility for participation in FRx interventions. Some healthcare providers screen patients for intervention placement by asking about their SES or food security status, others rely on a predetermined geographic location (typically food deserts), still others rely on health markers such as BMI or diabetes status ${ }^{(11-12,15,18,20-21,24)}$. Additionally, there is no standard in terms of which clinician delivers or manages the intervention. While some FRx interventions rely on a single physician as the sole administrator of prescriptions, other interventions rely on teams of healthcare providers, including nutritionists and dieticians, community health workers and assistants, registered nurses, pharmacists and pantry managers, to provide $\mathrm{FRx}^{(12,20)}$. Other important barriers include the lack of nutrition education or training for most clinical providers, clinic workflow issues and the need for additional staff for support ${ }^{(11,16,22,18)}$. There is also a general lack of discussion of the economic viability of FRx interventions ${ }^{(25)}$.

While the literature provides some insight into what FRx interventions in clinical settings do not have - standardisation, training and support - the literature does not provide any evidence on how this might impact a physician or clinic's decision to participate in or implement FRx interventions. Given the evidence for the clinical value of FRx interventions, the identified barriers to success and the role traditionally played by various healthcare providers in the rollout of these interventions, a better understanding of healthcare providers' perspectives is a key factor to understanding which FRx intervention components should be addressed systemically to develop a standard of practice for scaled implementation. This scaled implementation and standardisation is a critical first step in the financial sustainability of FRx interventions that, to date, are not reimbursable by third party payors.

The current study examines the general awareness of and attitude towards FRx among clinicians. Specifically, healthcare professionals were asked to articulate their level of comfort with nutrition advocacy, willingness to utilise a FRx and who they thought would most benefit from such an intervention. Additional questions examined the logistics of FRx implementation, specifically how patients would be screened for enrollment and if the currently available screening tools were adequate. FRx is an emerging approach in clinical care to addressing the system level drivers of obesity, diabetes and other chronic, nutrition-related conditions. However, there are few studies that have examined how clinicians and other healthcare providers involved in primary care perceive these interventions and little is known about the level of awareness of the logistics and the interventions' potential influence on patient outcomes. Further, studies that examine the usefulness of FRx interventions have an urban bias, with relatively few interventions being implemented in rural spaces ${ }^{(1-18)}$. Little is currently known about the potential for these interventions in rural settings, which often lack the clinical staff for intervention implementation, specifically those with formal nutrition science training, or the infrastructure for food procurement, such as farmers' markets ${ }^{(1-18)}$. This research addresses, in part or in full, each of these gaps by collecting the perspectives of rural clinicians on FRx interventions.

\section{Method}

The current study employed an exploratory research design using in-depth semi-structured interviews. A list of healthcare providers from North and Central Mississippi was generated through an online search for primary care facilities, family practice offices and obesity clinics in North Mississippi as well as through contacts in the region who knew clinics familiar with FRx interventions. Because this region is rural there are relatively few healthcare providers so the sample was pulled from a comprehensive list of all providers who served the areas and had available contact information online. These providers serve predominantly 
low-income communities. The initial sample included fifty physicians, registered dieticians and nurse practitioners, twelve from Oxford, MS, fifteen from Tupelo, MS, two from Batesville, MS, eight from Jackson, MS, nine from Charleston, MS, two from Grenada, MS, one from Cleveland, MS and one from New Orleans, LA. Each healthcare provider was contacted a total of six times, three times over the phone and three times via email. Lack of response after the sixth contact attempt was considered refusal to participate. Only twenty of the healthcare providers contacted responded to our request for an interview. Of the twenty healthcare providers who responded, two stated that they did not have time to participate; two stated that they did not want to be part of the study, but did not provide a reason and one stated that they did not want to be part of the study because they no longer worked in healthcare. The study sample included fifteen healthcare providers, including three physicians, four registered dieticians, three nurses and three nurse practitioners. Six interviewees, four of whom have used FRx, indicated familiarity with the concept. Nine interviewees indicated no previous knowledge of FRx. Healthcare providers in this sample were predominately white ( $n 14)$ and female ( $n 12)$. Participants in the same ranged from 26 to 38 years old and had a range of 3-27 years in their current profession, with a majority having over 10 years of experience. Notably, while all participants were associated with primary care facilities, they were not all primary care providers.

Interviews were conducted over the phone and were audio-recorded with the participants' consent. All interviews were de-identified and appropriate measures taken to protect the identity and privacy of the research participants. The research design and interviewing protocol were approved by the University of Mississippi Institutional Review Board as exempt under 45CFR 46·101(b)(\#2ii,iii) protocol number 20x-048.

\section{Analysis}

This research used summative content analysis and simultaneous coding to refine and synthesise the interview transcripts $^{(26)}$. Interview questions targeted four key areas of inquiry: (1) barriers to implementation, (2) potential use, (3) routinising on-boarding and (4) nutrition education and advocacy. Prior to analysis, keywords within each of these areas, or themes, were identified. For example, within the theme 'barriers to implementation', researchers identified five a priori codes (keywords), based on the FRx, to help analyze the data: compliance, nutrition knowledge, access to F \& V, clinic workflow and staffing. In order to fully capture all barriers to implementation, an additional category of 'other' was specified and codes pulled from the transcripts. Coding was conducted by three researchers using the predetermined coding protocol. The overall between-coder agreement was $81 \%$. Fleiss's $\kappa$ was also calculated for each interview and all had a positive inter-rater reliability (range: $0 \cdot 255-0 \cdot 927$ ).

\section{Results}

While healthcare providers in this sample did worry about their patients being able to follow-through on their FRx, the most common concerns with the efficacy of this approach were (1) a lack of understanding by healthcare providers of what a FRx is, how it is used and the logistics of administering such a prescription and (2) the cost of food (Table 1). Healthcare providers shared a pervasive concern that even if they could get doctors to write Rx the actual mechanics of such a clinical activity would be a significant barrier and their patients could not afford the food they would prescribe. For example, one participant felt it would be hard to get 'provider[s] [who] will actually write the script if it's an actual prescription'.

While not initially coded as a barrier, healthcare providers were asked about their use of screening tools to enroll patients in a FRx interventions and what they would change about existing tools they may already use. Overwhelmingly, participants, if they used a screening tool (10/15), used some objective health measures (7/10) - lab results, anthropometric assessments (BMI, weight) or the presence of a chronic condition. One healthcare provider mentioned specifically the 'starting a conversation diet screener' recommended by the American Medical Association, and the others used SES/income. Those not directly involved with primary care relied heavily on the referral of a primary care provider and did not use screening tools in their practice. Six providers mentioned a need to adjust their screening tool or protocol. These changes indicated a need to have some form of scorable index for need, extra personnel to do the assessment (i.e., 'diet tech'), the inclusion of other health measures ('mental health') or a need to waive current requirements for participation if multiple morbidities are present.

One healthcare provider articulated that it would be helpful to have 'somebody like a diet tech or a diet aid or a diabetes assistant to do a little bit more in-depth questioning before you send the educator or the clinician in'. Another noted that in their intervention they used an income-based screening process but wished they could 'make a waiver for those patients, maybe they have one or two comorbidities, and make that be the primary screening process ...'

Participants were also asked to envision the target population for a FRx intervention (Table 2). Most (8/15) indicated that patients with diabetes would be the target population, but patients with obesity or other chronic illnesses and patients with low SES were also identified (7/ 15). A few participants also mentioned targeting patients with pre-diabetes and those who lived in rural areas or food deserts.

While not all the participants agreed on the 'how' of routinising FRx intervention onboarding, all agreed that additional training, education and advocacy were key to successful implementation (Table 3). Several mentioned 
Table 1 Theme: potential barriers to implementation

\begin{tabular}{|c|c|c|}
\hline Keyword & Text examples & Count \\
\hline Patient compliance issues & $\begin{array}{l}\text { 1. 'I think [patients]... still [have] a little bit of skeptical thinking around like how is this really } \\
\text { going to help them' } \\
\text { 2. 'Another barrier I think it just going to be cost, so we're looking at are people going to be willing to } \\
\text { pay for food like they're willing to pay for prescriptions' } \\
\text { 3. 'sometimes the patients are just not compliant with it you know we give them the prescriptions' } \\
\text { 4. 'Pt's willingness to comply to a food rx' }\end{array}$ & 4 \\
\hline Nutrition knowledge & $\begin{array}{l}\text { 1. 'a barrier would of course be knowledge of nutrition and how to implement that' } \\
\text { 2. 'knowledge deficit' } \\
\text { 3. 'patients don't always know how to ... cook with fruits and vegetables' }\end{array}$ & 5 \\
\hline Access to $F \& V$ & $\begin{array}{l}\text { 1. 'the customers or the patients who may want the services for the food prescriptions may not } \\
\text { qualify.' } \\
\text { 2. 'getting local producers to agree to this implementation and the price of fresh fruits and veggies' }\end{array}$ & 2 \\
\hline Clinic workflow & No responses & 0 \\
\hline staffing & No responses & 0 \\
\hline other & $\begin{array}{l}\text { Transportation; getting physicians to write Rx; awareness; understanding; qualifying folks; cost } \\
\text { of food; feasibility with retailers; logistics }\end{array}$ & 8 \\
\hline
\end{tabular}

Table 2 Theme: potential use (of FRx)

\begin{tabular}{ll}
\hline Keyword & Text examples \\
\hline Programming & $\begin{array}{l}\text { 'I think that you could do cooking classes where people came to class and you could show them how to } \\
\text { cook certain things, which could take away the barrier of people not knowing how to use fruits and vege- } \\
\text { tables... So, you know maybe like a cooking demonstration where you could show them how to do it } \\
\text { and then they would also get like a box of food to go along with it.' }\end{array}$ \\
$\begin{array}{l}\text { No responses } \\
\text { Therapeutic }\end{array}$ \\
$\begin{array}{l}\text { 1. 'As a medicine. That understanding the value of a food prescription, that food is a medicine. It can be, it } \\
\text { can cure you' }\end{array}$ \\
$\begin{array}{l}\text { 2. 'I work a lot with people with diabetes and obesity and I have tons of clients who have been able to } \\
\text { completely come off all of their medicine by eating the right foods and, so, at the end they're actually } \\
\text { saving money because they're not buying this expensive diabetes medications and things of that nature, but } \\
\text { I would use them in my practice to be able to give people direction' }\end{array}$ \\
$\begin{array}{l}\text { 1. 'food is medicine in my opinion. I think that, you know, especially nursing- the foundation of nursing is } \\
\text { about holistic care - mind, body, soul. Food... makes up everything about those foundational princi- } \\
\text { ples' }\end{array}$ \\
$\begin{array}{l}\text { 2. 'anybody with especially diabetes or kidney disease or congestive heart failure, those three I would } \\
\text { definitely make sure that they had a food prescription because it has so much to do with their salt } \\
\text { intake, carbohydrates, things like that that affect their medication as well.' }\end{array}$ \\
\hline
\end{tabular}

the need for training across disciplines (nurse, RD, NP, physician), the hiring of specialised staff to assist with on-boarding (population health nurse), weekly or monthly educational seminars and the need for a state or federal agency to take on the responsibility and cost of systemic training. Healthcare providers in the current study also mentioned specific topics where more education was needed, including (1) types and use of screening tools, (2) community education, (3) potential benefits of such an intervention and (4) intervention logistics.

Fewer than half of the healthcare providers felt comfortable with their role in nutrition education and advocacy with patients (Table 4). Notably of the others who were comfortable, most had some training in nutrition (i.e., registered dietitian, had nutrition seminar in medical school). However, all but three participants said they would feel comfortable prescribing more fruits and vegetables.

\section{Discussion}

The results from the current study highlight three important findings related to healthcare providers' perceptions and attitudes towards FRx interventions. First, there is a systemic lack of understanding or awareness of FRx interventions. Most interviewees were not personally aware of FRx interventions or reported a concern that many in the field were unaware. While FRx interventions are not a new idea, they are relatively new to clinical settings. A comprehensive review of the literature suggests that these interventions happen in the local food arena - more specifically at farmers' markets and are usually funded by research bodies or federal agencies (i.e., USDA). While physicians often make the recommendations to their patients to increase fruit and vegetable intake or improve their diet generally, they are not typically involved in the administration 
Table 3 Theme: routinising on-boarding

\begin{tabular}{|c|c|c|}
\hline Keyword & Text examples & Cour \\
\hline Med-school training & $\begin{array}{l}\text { 1. 'Making sure training is more streamlined or more consistent among all disciplines' } \\
\text { 2. 'I think starting in school... . That's where you start, and then implementing it with, you know, } \\
\text { seasoned professionals' } \\
\text { 3. 'Education, so people know it's an option ... It would be so amazing if we had the nutrition, and } \\
\text { some schools are doing it, if we had it in school as a class absolutely. Because then you have } \\
\text { that underlying base and then you could do workshops to reiterate it when people get out and they } \\
\text { forget what they learned in school' } \\
\text { 4. 'I think it needs to start in medical school I really do. Because a lot of people are so used to just } \\
\text { treating what the problems are' } \\
\text { 5. 'It would probably be best to have another physician who is educated on this and already put it into } \\
\text { his/her practice to give lectures or individual education on the topic to med student and residents.' }\end{array}$ & 6 \\
\hline Continuing education units & $\begin{array}{l}\text { 1. 'part of their CEE's, their continuing education requirements' } \\
\text { 2. 'I think through continuing education would be good' } \\
\text { 3. 'Absolutely continuing education would be wonderful because we all have to have continuing } \\
\text { education, so I would definitely take that' } \\
\text { 4. 'continuing education but I think once doctors are already in practice, doing something like where } \\
\text { you would go to - I think that most physicians would find benefit in this as long as it would be no } \\
\text { extra work for them. So, I think that I mean just contacting physicians' offices would be one way, and } \\
\text { explaining how that would be done' }\end{array}$ & 5 \\
\hline $\begin{array}{l}\text { Hospital level professional } \\
\text { development }\end{array}$ & No responses & 0 \\
\hline $\begin{array}{l}\text { Clinic level professional } \\
\text { development }\end{array}$ & $\begin{array}{l}\text { 'I would think the best way would be through conventions, or certain people who are trained for } \\
\text { these types of programs. They could go, kind of like how the pharmaceutical companies, they'll } \\
\text { come and they'll bring lunch and they listen to about, you know, why you should use this drug. } \\
\text { That would be a very good way to get seasoned doctors and nurse practitioners on board with } \\
\text { this new tool' }\end{array}$ & 1 \\
\hline Hospital level policy change & No responses & 0 \\
\hline Clinic level policy change & No responses & \\
\hline State board certification & $\begin{array}{l}\text { 'maybe a certification like I said. So if I say oh this person has this, let me go to my reference and } \\
\text { say this is specifically what I would order just as I would look up a medication and say this is } \\
\text { what you will take and this is how many times a day you will take it and this is why you're going } \\
\text { to take it' }\end{array}$ & 1 \\
\hline National certification & No responses & 0 \\
\hline
\end{tabular}

of or activities associated with FRx interventions. Additionally, the literature offers up a wide variety of definitions for FRx interventions. Many of the interviewees could not supply a clear definition and were only able to talk about FRx interventions within the context of their practice after researchers provided a definition.

Second is the need for systemic combination screening tools. While a range of screening tools was mentioned by healthcare providers, two things were evident: (1) there was a significant reliance on biometrics as a screening tool, but no consistency across providers about which ones to use and (2) there was very little consideration of non-biometric assessments. Importantly, there was no mention of food security status or the use of the USDA two-item, six-item or long-form assessment of food insecurity as a screening tool. FRx are designed not only to address the nutritional deficiencies in a patient's diet but also to improve access to food more generally. A number of participants made note of this nuance and indicated a need for being more sensitive to SES and other comorbidities. This highlights the need for a multifaceted screening tool that allows healthcare providers to screen for the health-related pre-conditions for participation but also food security status and/or income that might make a patient a more compelling candidate for participation in a FRx intervention.
Third is the need for comprehensive, interdisciplinary training programmes for healthcare providers. Patients do not engage with just one type of healthcare professional, rather they engage with an entire healthcare system which can include a multitude of healthcare professionals. Specific training in nutrition is needed across all types of providers. Many of the study respondents identified medical school as a good opportunity to implement nutrition education. The Goldring Center for Culinary Medicine at Tulane University is one such example. Students in this programme learn about food and nutrition in combination with their traditional medical training ${ }^{(27)}$.

A sample size of $25-30$ is ideal for qualitative work, especially grounded research studies ${ }^{(28-29)}$. However, in exploratory studies, like this one, where the goal is to develop a base understanding of the phenomenon details (e.g. awareness and attitudes toward FRx interventions) rather than determine how frequent the phenomenon is within the population, $<20$ interviews is appropriate ${ }^{(30)}$. As few as twelve interviews can result in data saturation during qualitative work $^{(31)}$. Despite a small and nonrepresentative (of the whole US) sample was used, the following recommendations are based on the fine-grained details of how healthcare providers interpret, understand and relate to FRx interventions. The following recommendations provide a starting place for improving healthcare 
Table 4 Theme: nutrition and health advocacy

\begin{tabular}{|c|c|c|}
\hline Keyword & Text examples & Count \\
\hline $\begin{array}{l}\text { Comfort with nutrition } \\
\text { advocacy }\end{array}$ & $\begin{array}{l}\text { 1. 'I'm a registered dietician with } 20 \text { years of experience, so I'm pretty confident with... nutrition } \\
\text { education' } \\
\text { 2. 'On a scale of } 1-10 \text { a } 10 \text {. On a scale of } 1-100,100 \text { ' } \\
\text { 3. 'I feel pretty comfortable with nutrition... as far as being an advocate for it l've been a nurse for } \\
20 \text { years, l've seen what good nutrition can do for people' } \\
\text { 4. 'on a scale of } 1-10 \text {, it's an 8' } \\
\text { 5. 'I think l'm pretty comfortable, I'm not an expert but I think I'm pretty comfortable' } \\
\text { 6. 'I'm a dietician so l'm pretty comfortable with that' } \\
\text { 7. 'Extremely comfortable' } \\
\text { 8. 'I have a huge level of comfort with it' } \\
\text { 9. 'That's what I do, so l'd say real, real comfortable' }\end{array}$ & 6 \\
\hline
\end{tabular}

Would use a FRx $\quad$ 1. 'Yeah, so I'm of course very comfortable in writing food prescriptions, and one of the reasons why l'm so comfortable with that is because we have to equip people with actionable items when they leave our office... we really have to help people operationalize that and so, you know, writing a food prescription that says "dark greens" and examples of what those are, you know, half a cup cooked or a full cup raw twice a day. That gives someone much more information about how to incorporate that into their diet versus just telling people to eat more green leafy vegetables'

2. 'Oh, definitely. Yeah, for sure because I believe in food, using food in this manner and l've seen it in my own practice daily where the right foods really do heal and the wrong foods really do harm. And I think just another reason why is I've seen the reciprocity on behalf of the patient when I say "hey," like, even with exercise, you know "let's get 30 minutes of exercise every day" versus just saying "you know it'd be good if you exercised," if you have like a tangible item in their hand, especially I find for the people of a lower health literacy, they really need guidance and instruction and if you literally hand them a piece of paper that says what to do they're much more likely to do it especially if it's coming from a place of authority like that'

3. 'Yes, actually. Especially when it comes to the three big diagnoses of congestive heart failure, diabetes, and kidney dysfunction, and those specific, you know, whatever contributes to their kidneys where, if it's salt or carbohydrates, which would be diabetes and things like that. And high cholesterol. So, in those populations l'd say l'm $90 \%$ confident but, yes, l'd use food prescriptions for those things'

4. 'Yes, I do. I think, like I said, because I'm a member of the wellness center and I have attended a lot of the classes that they have and participated in a lot of the challenged that they have and as a participant in those challenges, part of it is going to those classes so because of that I think I'm pretty knowledgeable as far as what are the risk factors, what are the foods that I need to recommend that I eat myself and also for my patients to eat. So, I think I'm pretty knowledgeable'

5. ' $100 \%$. And why? Because everything is about what we put in our bodies, so I think this is the most important thing that we could be doing for our patients. I'm very excited to hear about this.'

6. 'Absolutely. Just because I know that our providers are getting better at um who needs it and better at how they can use this to benefit the community, so yeah I'm confident in them and their ability to do this'

7. 'Yes. I mean it's something that everybody needs. Everybody needs nutrition and a good source of food and so I would be comfortable with it'

8. 'I would if I had the education about it because that's the thing about it, I mean I don't like people writing prescriptions unless I know for sure exactly what needs to be done in a proper way. So much is regulated about us in what we can and cannot do and having someone to explain it to us and what options are, and then someone helping us to do it after that would be more likely to be of benefit'

9. 'Yes because I mean l've already done things similar to that before, so.'

providers' ability to use and implement FRx interventions in clinical settings to improve health outcomes for vulnerable patients.

\section{Recommendations}

Based on these findings, the authors have developed three recommendations for improving clinical implementation of FRx for the treatment of chronic conditions:

1. Screening standardisation. Healthcare professionals and healthcare researchers should look to the standardisation and validation of a comprehensive screening tool for enrolling patients into FRx interventions (or similar interventions designed to increase nutrient food consumption). The current study indicates that healthcare providers are seeking tools that combine biometrics with items that help them prioritise patients according to SES and potential benefits to health status. To date, there are a few tools that provide a good foundation for this process. The 'starting the conversation' or STC ${ }^{(32)}$ assessment performs well for assessing diet quality, as does the CDC's BRFSS ${ }^{(33)}$. If combined with existing tools for assessing food insecurity, such as the USDA's two- or six-item screening tool ${ }^{(34)}$, and biometric measures that proxy for long-term morbidities and quality of life, the STC or BRFSS could 
provide a comprehensive screening tool for providers. Future work should focus on the validation of a combination tool, specifically as it relates to the enrollment of patients in FRx interventions. Until such a tool becomes available, in those places where nutrition education is important, advocating a multifaceted approach to screening is important.

2. Multi-tiered training across disciplines. This training should take place across three arenas: (1) medical or nursing school, (2) continuing education units and (3) a national certifying mechanism. Offering training during initial training and as continuing education units allows healthcare professionals to develop a foundational knowledge and maintain that knowledge over the course of their career. A national certifying mechanism allows professionals who did not receive training in medical or professional school to access that training to better serve their patients. This training should include basic nutrition education, the role of FRx interventions in improving patient outcomes, information and training on screening tools and information on intervention logistics (how to set up, implement and evaluate FRx interventions) ${ }^{(35)}$. To be clear, this approach is not meant to replace certified nutrition therapies as prescribed by registered dietician or advocate for clinicians becoming RD alternatives, but rather to initiate these conversations with patients, direct them to the proper care and provide helpful information.

3. Community advocacy training. Community advocacy is not a part of healthcare training, but clinicians are at the front lines of many healthcare problems. Providing medical professionals with an advocacy framework and the skills to articulate their patients' concerns to a broad public and policy audience is crucial $^{(36)}$. In part, serious efforts to promote advocacy by healthcare providers start with including this type of education in professional schools and rethinking how students are selected for these training programmes. To move advocacy to the forefront, medical training programmes need to overcome the current emphasis on biomedical knowledge and equally emphasise activities that demonstrate skills related to social justice issues ${ }^{(37)}$. To facilitate the inclusion of advocacy in both initial training and continuing education, it is helpful to consider potential frameworks for health advocacy related to nutrition. University of British Columbia Health Advocacy Framework articulates the role or roles (agency $v$. activism) providers can have in advocacy and the nature of that advocacy (shared $v$. directed) ${ }^{(38)}$. This model allows healthcare providers to choose from a range of advocacy activities that match the needs of their community, as well as their expertise. Additionally, the Community Health Worker Advocacy Framework, while originally developed for community health workers, readily translates to the work of primary healthcare providers. This framework provides the scaffolding for an iterative, reflective advocacy strategy ${ }^{(39)}$. Both of these frameworks could be adapted to apply specifically to nutrition and environmental health.

\section{Conclusion}

The current study sought to explore healthcare professionals' attitudes towards and awareness of FRx interventions. While the healthcare professional interviewed for the current study was generally supportive, findings revealed a widespread lack of knowledge of what FRx are and how they work. However, most healthcare providers agreed that FRx would benefit their patients with chronic metabolic conditions like diabetes and hypertension, as well as those with lower SES. Although exploratory, the current study has several implications for healthcare praxis. First, there is a growing need and desire for interdisciplinary approaches to nutrition within the scope of traditional healthcare practice. The increasing prevalence of chronic metabolic disorders makes multipronged approaches that include traditional medicinal therapies AND interventions that improves access to and knowledge of healthy foods essential. These multipronged approaches require a common understanding of nutrition and health and many healthcare providers lack this training. Additionally, there needs to be consistency across healthcare settings in how patients are enrolled in these types of nutrition/food access interventions. To fully understand the scale and level of impact on health outcomes, consistence in patient profiles is important. This requires validated screening tools that account for current health status and SES.

\section{Acknowledgements}

Acknowledgements: The authors would like to acknowledge our community partner Dr. Catherine Moring who assisted with recruitment. The authors would also like to thank the healthcare providers who agreed to participate in the current study. Each of the authors would also like to thank their respective departments for their continued support. Financial support: This work was not supported through external or internal funding. Conflict of interest: The authors have no conflict of interest as it relates to the content of this manuscript. Authorship: Each author has contributed significantly to the writing, analysis and editing of this manuscript. K.B.C. was responsible for conducting interviews, completing transcriptions and leading analysis. She also co-led the drafting of the introduction. Drs K.B.C and M.R. were responsible for conceptualisation, research design, quality control of data and analysis. Dr A.C. was responsible for drafting the results and discussion. 
Dr M.R. was responsible for editing of the final manuscript. D.A. and Q.P. were responsible for data analysis, drafting the introduction and editing of the final manuscript. Ethics of buman subject participation: The current study was conducted according to the guidelines laid down in the Declaration of Helsinki and all procedures involving human subjects/patients were approved by the University of Mississippi Institutional Review Board. Verbal informed consent was obtained from all subjects/patients. Verbal consent was witnessed and formally recorded. The research team adhered to all the protocols approved through the University of Mississippi IRB (20X-048).

\section{References}

1. AbuSabha R, Namjoshi D \& Klein A (2011) Increasing access and affordability of produce improves perceived consumption of vegetables in low-income seniors. $J$ Am Dietetic Assoc 111, 1549-1555.

2. Berkowitz SA, Terranova J, Hill C et al. (2018) Meal delivery programs reduce the use of costly health care in dually eligible Medicare and Medicaid beneficiaries. Health Affairs 37, 535-542.

3. Bryce R, Guajardo C, Ilarraza D et al. (2017) Participation in a farmers' market fruit and vegetable prescription program at a federally qualified health center improves hemoglobin A1C in low income uncontrolled diabetics. Prev Med Rep 7, 176-179.

4. Chrisinger A \& Wetter A (2016) Fruit and vegetable prescription program: design and evaluation of a program for families of varying socioeconomic status. J Nutr Educ Behav 48, S57.

5. Freedman DA, Bell BA \& Collins LV (2011) The Veggie Project: a case study of a multi-component farmers' market intervention. J Prim Prev 32, 213.

6. Hossfeld L, Kerr LJ \& Belue J (2019) The Good Food Revolution: building community resiliency in the Mississippi Delta. Soc Sci 8, 57.

7. Kass E \& Kolasa K (1978) Supplemental commodity food use by participants in the project focus: hope in Detroit, Michigan. Ecol Food Nutr 7, 57-61.

8. Marcinkevage J, Auvinen A \& Nambuthiri S (2019) Washington state's fruit and vegetable prescription program: improving affordability of healthy foods for low-income patients. Prev Chronic Dis 16, E91.

9. Savoie-Roskos M, Durward C, Jeweks M et al. (2016) Reducing food insecurity and improving fruit and vegetable intake among farmers' market incentive program participants. J Nutr Educ Behav 48, 70-76.

10. Wetherill MS, McIntosh HC, Beachy C et al. (2018) Design and implementation of a clinic-based food pharmacy for food insecure, uninsured patients to support chronic disease self-management. J Nutr Educ Behav 50, 947-949.

11. Goddu AP, Roberson TS, Raffel KE et al. (2015) Food Rx: a community-university partnership to prescribe healthy eating on the South Side of Chicago. J Prev Intervention Commun 43, 148-162.

12. Hess A, Passaretti M \& Coolbaugh S (2019) Fresh food farmacy. Am J Health Promot 33, 830-832.

13. Ridberg RA, Bell JF, Merritt KE et al. (2019) A pediatric fruit and vegetable prescription program increases food security in low-income households. J Nutr Educ behav 51, 224-230.

14. Saxe-Custack A, LaChance J \& Hanna-Attisha M (2020) Child consumption of whole fruit and fruit juice following six months of exposure to a pediatric fruit and vegetable prescription program. Nutrients $\mathbf{1 2}, 25$.

15. Cavanagh M, Jurkowski J, Bozlak C et al. (2017) Veggie Rx: an outcome evaluation of a healthy food incentive programme. Public Health Nutr 20, 2636-2641.

16. Forbes JM, Forbes CR, Lehman E et al. (2019) "Prevention Produce": integrating medical student mentorship into a fruit and vegetable prescription program for at-risk patients. Permanente J 23, 18-238.

17. Kearney M, Bradbury C, Ellahi B et al. (2005) Mainstreaming prevention: prescribing fruit and vegetables as a brief intervention in primary care. Public Health 119, 981-986.

18. Joshi K, Smith S, Bolen SD et al. (2019) Implementing a produce prescription program for hypertensive patients in safety net clinics. Health Promot Pract 20, 94-104.

19. Peek ME, Ferguson MJ, Roberson TP et al. (2014) Putting theory into practice: a case study of diabetes-related behavioral change interventions on Chicago's south side. Health Promot Pract 15, 40S-50S.

20. Anand S (2014) Prescribing prevention: fresh fruits and vegetables are just what the doctor orders. Contemp Pediatr 31, $5-7$.

21. Burrington CM, Hohensee TE, Tallman N et al. (2020) A pilot study of an online produce market combined with a fruit and vegetable prescription program for rural families. Prev Med Reports 17, 101035.

22. Schlosser AV, Smith S, Joshi K et al. (2019) "You Guys Really Care About Me...": a qualitative exploration of a produce prescription program in safety net clinics. $J$ General Intern Med 34, 2567-2574.

23. Smith S, Malinak D, Chang J et al. (2017) Addressing food insecurity in family medicine and medical education. Fam Med 49, 765-771.

24. Swartz H (2018) Produce Rx programs for diet-based chronic disease prevention. AMA J Ethics 20, 960-973.

25. Sadler K, Bontrager E, Rogers B et al. (2012) Food by Prescription: Measuring the Impact and Cost-Effectiveness of Prescribed Food on Recovery from Malnutrition and HIV Disease Progression among HIV+ Adult Clients in Ethiopia. Boston: Feinstein International Center, Tufts University.

26. Hsieh H \& Shannon SE (2005) Qualitative health research. Qual Health Res 159, 1277-1288.

27. McNulty I (2018) Tulane Med Students Learn to Cook for Their Patient's Sake. The Advocate New Orleans, 7 June. https://medicine.tulane.edu/news/tulanemed-students-learn-cook-their-patients-sake (accessed July 2020).

28. Charmaz K (2017) Constructing Grounded Theory: A Practical Guide Through Qualitative Analysis. London: Sage Publications.

29. Morse JM (1995) The significance of saturation. Qual Health Res 5, 147-149.

30. Crouch M \& McKenzie H (2006) The logic of small samples in interview-based qualitative research. Soc Sci inform $\mathbf{4 5}$, 483-499.

31. Guest G, Bunce A \& Johnson L (2006) How many interviews are enough? An experiment with data saturation and variability. Field methods 18, 59-82.

32. Paxton AE, Strycker LA, Toobert DJ et al. (2011) Starting the conversation: performance of a brief dietary assessment and intervention tool for health professionals. Am J Prev Med $\mathbf{4 0}$, $67-71$.

33. Centers for Disease Control and Prevention (2011) Behavioral Risk Factor Surveillance System Survey Questionnaire. Atlanta, GA: US Department of Health and Human Services, Centers for Disease Control and Prevention.

34. United States Department of Agriculture Economic Research Service (2012) US household food security 
survey module: Six-Item short form, 1 September. https:// www.ers.usda.gov/media/8282/short2012.pdf (accessed July, 2020).

35. National Institutes of Health (2019) Implementation science news, resources and funding for global health researchers. Fogarty International Center, 1 May. https://www.fic.nih. gov/ResearchTopics/Pages/ImplementationScience.aspx (accessed July 2020).

36. Luft LM (2017) The essential role of physician as advocate: how and why we pass it on. Can Med Educ J 8, e109.
37. Mu L, Shroff F \& Dharamsi S (2011) Inspiring health advocacy in family medicine: a qualitative study. Educ Health 24, 534.

38. Hubinette M, Dobson S, Scott I et al. (2017) Health advocacy. Med Teacher 39, 128-135.

39. Sabo S, Ingram M, Reinschmidt KM et al. (2013) Predictors and a framework for fostering community advocacy as a community health worker core function to eliminate health disparities. Am J Public Health 103, e67-e73. 